\title{
The Modern Art of Reading Computed Tomography Images of the Lungs: Quantitative CT
}

\author{
Felix J.F. Herth ${ }^{a, b}$ Miranda Kirby ${ }^{c}$ Jered Sieren ${ }^{c}$ Jonas Herth ${ }^{b}$ Joshua Schirm ${ }^{c}$ \\ Susan Wood ${ }^{\mathrm{c}}$ Maren Schuhmann ${ }^{\mathrm{a}}$ \\ ${ }^{a}$ Department of Pulmonology and Critical Care Medicine, Thoraxklinik, and ${ }^{\mathrm{b}}$ Translational Lung Research Center \\ Heidelberg (TLRC), German Center for Lung Research, Heidelberg, Germany; ' VIDA Diagnostics, Inc., Coralville, IA, USA
}

\section{Keywords \\ COPD - Emphysema · Asthma - Lung cancer - Computed tomography - Quantitative computed tomography}

\begin{abstract}
Lung diseases are increasing in prevalence and overall burden worldwide. To stem the tide, more and more national and international guidelines are recommending the use of various diagnostic algorithms that are disease specific. There is growing consensus among the respiratory community that although patient histories and lung function testing are the minimum required for clinical examinations, these tests alone are not sufficient for disease characterization. Therefore, the use of computed tomography (CT) imaging is increasing used in clinical decision making for lung diseases. Lung diseases affect various components of lung, including the small airways, lung parenchyma, the interstitial space and the pulmonary vasculature. Quantitative CT (QCT) methods are emerging and are increasingly available using commercial software to quantify the underlying disease components, and a growing body of evidence suggests that QCT is an important tool in the clinical setting to help accurately and reproducibly detect where the disease is located in the lung, and to quantify the extent and overall severity for sev-
\end{abstract}

eral lung diseases. Furthermore, this growing body of evidence has promoted the use of thoracic QCT to the point that it is now considered by many as an indispensable technology for longitudinal analysis and intervention trials. Many QCT imaging measurements are available to the respiratory physician, and the aim of this review is to introduce and describe pulmonary QCT imaging measurements and methodologies.

(c) 2017 S. Karger AG, Basel

\section{Introduction}

The burden of respiratory diseases impacts not only the individuals, but also their families, schools, workplaces, neighborhoods, cities, and states. The number of patients diagnosed with respiratory diseases is rapidly increasing [1]. Respiratory symptoms are among the major causes of consultation at primary health-care centers [2].

Some lung diseases, like asthma and chronic obstructive lung disease (COPD), involve the narrowing or blockage of the airways resulting in poor air flow. Other lung diseases, including pulmonary fibrosis, pneumonia, and lung cancer, are caused by a loss of elasticity in the lungs that produces a decrease in the total volume of air

\section{KARGER}

(c) 2017 S. Karger AG, Basel

E-Mail karger@karger.com

www.karger.com/res
Felix J.F. Herth

Thoraxklinik, University of Heidelberg

Röntgenstrasse 1

DE-69126 Heidelberg (Germany)

E-Mail felix.herth@med.uni-heidelberg.de 
that the lungs are able to hold. Research has shown that long-term exposure to air pollutants can reduce lung growth and development and increase the risk of developing asthma, COPD, and other respiratory diseases [3].

In addition to the clinical examination, evaluating the patient history and lung function testing, most international disease-based guidelines also recommend imaging. Conventional measurements of lung function are only available at one location (the mouth) and therefore do not provide any regional information. Due to the rapid developments of computed tomography (CT) imaging technology, the dramatic reduction in the radiation burden associated with CT in recent years, and the clear diagnostic improvement of CT compared to classical chest $\mathrm{X}$-ray, CT has emerged as the gold standard imaging modality for lung disease evaluation. However, the information gained by CT imaging is overwhelming, therefore motivating the development of CT imaging analysis software to streamline quantitative CT (QCT) into clinical use.

The quantification of emphysema via lung density thresholding in CT images dates back more than 2 decades [4], and is now accepted for use as an outcome measure in studies of emphysema-modifying therapy in patients with alpha-1-antitrypsin deficiency [5-7]. CT technologies to assess the extent of airway disease have also been developed, and airway wall morphometry is used as a measure of airway remodeling in COPD and asthma [8-10]. Depending on the indications, various other QCT measurements are used by different groups worldwide to diagnose, stage, and control various lung diseases. Therefore, commercially available software solutions can provide quantitative imaging endpoints for assessing the impact of therapies on lung function. However, to facilitate earlier decisions regarding the impact of therapeutic treatments, standardized image acquisition protocols and quality certified and validated image analysis methodology is required to ensure high accuracy and precision of the quantitative information provided.

The purpose of this report is to define QCT measurements, provide evidence of their utility in certain applications where available, and to discuss the potential of these measurements in various applications.

\section{Techniques for QCT of the Lung}

Below, we have described a need for a standardized image acquisition combined with core lab analysis to obtain high quality and reproducibility images, and the QCT measurements that can be derived; a summary of all QCT measurements and their potential application in lung diseases is shown in Table 1.

\section{Standardizing Image Acquisition Protocols}

Accurate and reproducible analysis of the lungs using QCT metrics requires a standardized CT image acquisition protocol [11]. CT protocols are dynamic, and technology is rapidly advancing as CT systems are updated and lower-dose options become available $[12,13]$. It is important to those in industry providing QCT measures to adapt to these CT technology advancements, and understand how these advancements impact QCT measurements.

It is well-described that CT systems can have both global scanner Hounsfield unit (HU) calibration bias as well geometric and filtration differences, which may impact the density histogram, texture, tissue, and airway measures [14]. Although there are no formal standards yet for CT imaging of the lung, there are new working groups that aim to better understand and minimize this interscanner variability, such as the Quantitative Imaging Biomarker Alliance (QIBA) with the Radiological Society of North America (RSNA) [15]. The SubPopluations and Intermediate Outcome Measures In COPD (SPIROMICS) multi-site, cohort study has also recently outlined the best practice for QCT imaging for multi-site studies in which images were acquired using multiple CT scanner makes and models [16]; a standardized imaging protocol was detailed for various CT scanners, where spatial (1-mm slices), temporal resolution, use of a standard CTDvol across body sizes, and a particular reconstruction kernel for the various scanners was provided.

Standardizing image acquisition protocols to minimize nonbiological variability is critical for longitudinal and other serial studies, and for detecting early, subclinical disease changes. In this regard, it was recently demonstrated that using the SPIROMICS standardized protocol, as well as the same protocol and parameters at both baseline and follow-up, QCT was able to detect significant changes in COPD progression only 1 year after follow-up [16]. Another key paper employing the same standardized SPIROMICS image acquisition protocol identified a subgroup of smokers that had normal spirometry and no radiological signs of hyperinflation, but exhibited COPD symptoms [17]. QCT, however, detected very small (submillimeter) changes in airway wall thickness, further validating that disease-related changes are subtle, and may only be detected in studies that have robust CT standardized protocols. 
Table 1. Overview of QCT measurements and applications

\begin{tabular}{|c|c|c|c|c|c|c|}
\hline & COPD & Emphysema & $\begin{array}{l}\text { Chronic } \\
\text { bronchitis }\end{array}$ & Asthma & ILD & Cancer \\
\hline \multicolumn{7}{|l|}{ Density measurements } \\
\hline Low attenuation area (\%) & $\times$ & $\times$ & & & & \\
\hline Low attenuation cluster & $\times$ & $\times$ & & $\times$ & & \\
\hline LAC slope & $x$ & $x$ & & & & \\
\hline Max cluster size (\%) & $\times$ & $\times$ & & & & \\
\hline 15\% percentile (HU) & $x$ & $\times$ & & & & \\
\hline Air trapping (\%) & $\times$ & $x$ & & $\times$ & & \\
\hline \multicolumn{7}{|l|}{ Heterogeneity measurements } \\
\hline Heterogeneity (\%) & $x$ & $\times$ & & & & \\
\hline Heterogeneity weighted (\%) & $\times$ & $\times$ & & & & \\
\hline \multicolumn{7}{|l|}{ Tissue measurements } \\
\hline Tissue volume (mL) & $\times$ & $\times$ & & $\times$ & $\times$ & \\
\hline Lung tissue mass (g) & $x$ & $x$ & & $\times$ & $x$ & \\
\hline Thoracic volume $(\mathrm{mL})$ & $\times$ & $\times$ & & & & \\
\hline Disease probability map (\%) & $\times$ & $\times$ & & $\times$ & $\times$ & \\
\hline Fissure integrity (\%) & $\times$ & $\times$ & & & & \\
\hline \multicolumn{7}{|l|}{ Vascular measurements } \\
\hline Total vascular volume (mL) & $\times$ & $\times$ & $\times$ & & & \\
\hline Peripheral vascular volume (mL ) & $\times$ & $\times$ & & $\times$ & & \\
\hline Bronchoarterial ratio & $x$ & $x$ & $\times$ & $\times$ & & \\
\hline \multicolumn{7}{|l|}{ Airway measurements } \\
\hline Luminal diameter (max/min) & $\times$ & $\times$ & & $\times$ & & $\times$ \\
\hline Lumen diameter circle equivalent & $\times$ & & & $\times$ & & $\times$ \\
\hline Wall thickness (mm) & $x$ & $x$ & $\times$ & $\times$ & $x$ & \\
\hline Airway wall fraction & $\times$ & $x$ & $\times$ & $x$ & $x$ & \\
\hline Airway circularity & $\times$ & $\times$ & $\times$ & $\times$ & $x$ & \\
\hline Airway concaveness & $\times$ & $x$ & $\times$ & $x$ & $x$ & $\times$ \\
\hline $\operatorname{Pi} 10(\mathrm{~mm})$ & $\times$ & $x$ & & $x$ & $x$ & \\
\hline Airway labeling & & $\times$ & $\times$ & $x$ & $\times$ & $\times$ \\
\hline \multicolumn{7}{|l|}{ Airway path planning } \\
\hline Angle to target & & $x$ & & $\times$ & $\times$ & $\times$ \\
\hline Airway extension to target & & $\times$ & & $x$ & $x$ & $x$ \\
\hline
\end{tabular}

In addition to standardizing image acquisition protocols, QCT imaging software solutions can provide highly accurate and reproducible measurements. It is important to note that although fully automated QCT eliminates inter- and intrareader variation, high-quality QCT requires expert analyst verification, particularly in clinical applications where QCT may be used in diagnosis or impact treatment decisions. Therefore, combining a robust standardized QCT imaging protocol and ISO quality certified and validated image analysis methodology that includes expert analyst verification is considered the best practice for today's QCT imaging.

\section{Density Measurements}

Low Attenuation Areas. The low attenuation areas (LAA) is a measure of lung density, defined as the percentage of voxels in the lung below a certain threshold. Although numerous CT density thresholds have been investigated, the $-950 \mathrm{HU}$ threshold is most commonly used $[4,18]$. LAA $_{950}$ has been validated using histology and therefore serves as a surrogate measurement of emphysema [19]. An important advantage of QCT imaging is the ability to localize these LAA to specific regions of the lung (i.e., lobes and sublobes) as shown in Figure 1a. Another advantage of QCT measurements is the high
10
Herth/Kirby/Sieren/Herth/Schirm/Wood/ Schuhmann 


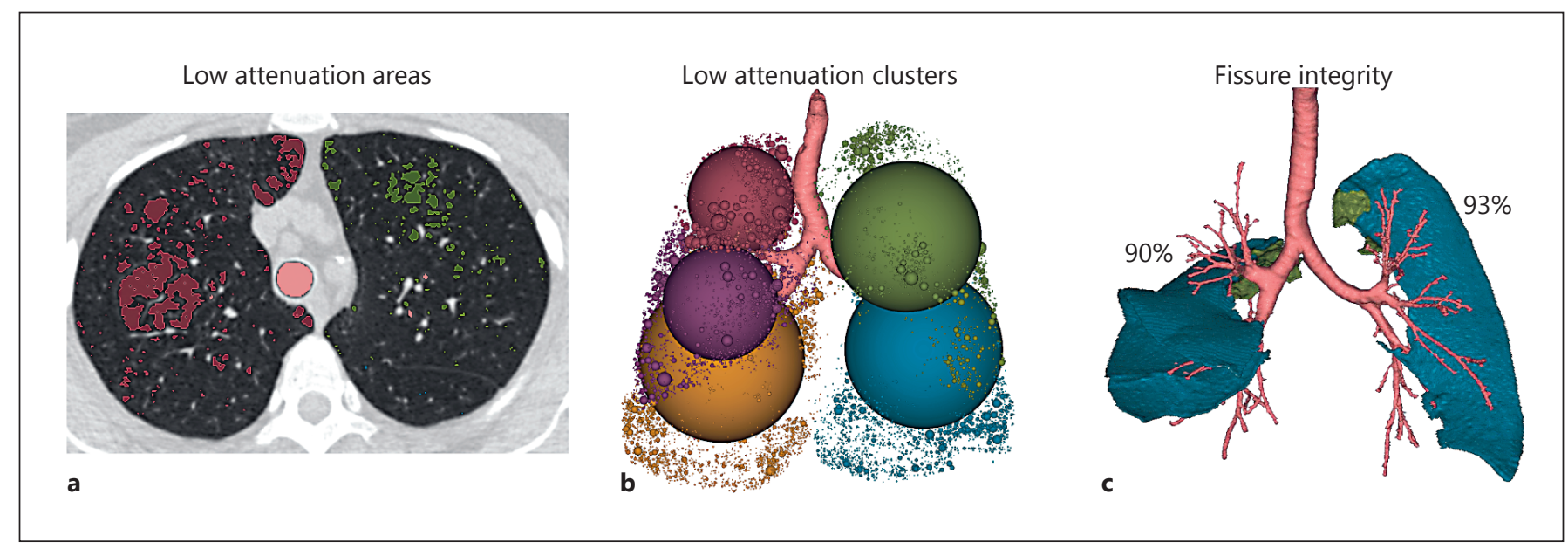

Fig. 1. QCT density measurements. a Low attenuation areas below $-950 \mathrm{HU}$ are highlighted in green for the right lung and red for the left lung (Apollo software; VIDA Diagnostics Inc., Coralville, IA, USA). b 3D representation of the low attenuation clusters below $-950 \mathrm{HU}$ (color coded for the different lung lobes) (Apollo software). c 3D representation of fissure completeness, where the green and blue areas represent the incomplete and complete portions of the fissure surfaces (Apollo software).

reproducibility. There is significant variability within and between observers for visual radiology assessments $[20,21]$. Studies have also suggested that visual scoring may underestimate emphysema extent in mild disease [18] and overestimate emphysema in more severe disease $[18,20]$. Furthermore, there is evidence to suggest that quantitative emphysema is better correlated with morphological measures of emphysema than visual scoring $[20,22]$.

High Attenuation Areas. The high attenuation areas (HAA) is a measure of the percentage of voxels in the lung between -600 and $-250 \mathrm{HU}$. HAA has been shown to be associated with cigarette smoking [23] and increased odds of interstitial lung abnormalities [24]. HAA has also been shown to be associated with biomarkers of inflammation, extracellular matrix remodeling, reduced lung function, and a higher risk of death [25].

Low Attenuation Cluster Analysis. Low attenuation cluster (LAC) provides a visual representation of emphysema as spheres or "clusters," as shown in Figure 1b. The clusters show lung regions defined by connected voxels below a specific threshold, grouped into spheres for ease of visualization. Using $\mathrm{CT}$ acquired at full inspiration and a LAA density threshold (e.g., $-950 \mathrm{HU}$ ), these clusters can indicate the size of the "emphysematous hole." These clusters can also indicate regions of gas trapping when performed using expiratory CT, and can be used globally or regionally to find LAA in specific regions of the lung (i.e., lobe or sublobe). Quantitative measurements derived from the LAC analysis are described below:

LAC Slope. Quantitative LAC analysis can be performed by plotting the number of LAA clusters against the size of clusters and taking the slope [26]. A steep slope indicates fewer "big clusters" or more small emphysematous holes, possibly indicating early disease or diffuse pan-lobular emphysema. Conversely, a less steep slope indicates more "big clusters." This analysis can be performed on the whole lung or regionally. It has been suggested that the LAC is less sensitive to other potential causes of low attenuation (i.e., image noise), and studies suggest that LAC correlates better with radiologist visual scoring than LAA [18].

LAC Max Cluster Size. This represents the volume of the largest "sphere" (low density area) in a given lung/ lobe, expressed as a percentage; the max cluster size indicates the location of the largest concentration of emphysema (e.g., lung lobe, sublobe).

15th Percentile. This is another well-established density measurement for estimating emphysema that uses fixed percentiles of the frequency distribution of CT density. Gould et al. [27] showed that the lowest 5th percentile correlated with distal airspace size taken in resected lung/lobe from patients that underwent resection for a peripheral lung tumor. More recently, a study by Dirksen et al. [6] in patients with severe alpha-1 antitrypsin deficiency concluded that the decline in CT density percentile 
may provide a sensitive measure of emphysema progression. This has led to the recommendation of the lowest 15 th percentile as a measurement of emphysema and emphysema progression $[28,29]$.

Air Trapping. Air trapping provides a percentage of LAA on expiratory CT images, thought to represent hyperinflation or "air trapping," by location (e.g., lung, lobe, sublobe). Air trapping is a measure of lung density on CT images acquired at full-expiration, defined as the percentage of voxels below a given threshold; several thresholds have been investigated, including the $-856 \mathrm{HU}$ threshold cutoff on end-expiratory CT images [30], the $-850 \mathrm{HU}$ [8] and the $-900 \mathrm{HU}$ [31] threshold, although the $-856 \mathrm{HU}$ threshold is most commonly used. CT air trapping measurements are significantly correlated with measures of pulmonary function in COPD [32] and asthma [30, 31, 33], and can be used to assess response to therapy [34].

Fissure Integrity. The percentage of the fissure surface with a complete fissure can be measured on CT images [35]. Figure $1 \mathrm{c}$ shows a 3D representation of fissure completeness (Apollo software; VIDA Diagnostics Inc., Coralville, IA, USA), where the green and blue areas represent, respectively, the incomplete and complete portions of the fissure surfaces. Fissure integrity has been validated as a minimally invasive surrogate for collateral ventilation [36], which is critical in endoscopic treatment selection for severe emphysema patients.

\section{Heterogeneity Measurements}

Emphysema Heterogeneity. The emphysema heterogeneity score is a surrogate measure of the distribution of emphysema in the lung and is considered an important indicator of success when treating emphysema with lung volume reduction devices (e.g., one-way valves). Patients with visual evidence of an upper lobe, heterogeneous pattern of emphysema have been associated with improved outcomes following lung volume reduction surgery [37]. Quantitatively, emphysema heterogeneity has been defined as the difference in the LAA (below -910 HU [38] or $-950 \mathrm{HU}$ [36]) between the targeted lobe and the ipsilateral adjacent nontarget lobe, and greater heterogeneity of emphysema between lobes has been shown to identify patients with greater functional and physiological responses to endobronchial valve therapy [38]. Another study, however, indicated that patients with evidence of complete fissure and lobar occlusion benefited from endobronchial valve treatment, independent of emphysema heterogeneity [39].

Heterogeneity Weighted. The heterogeneity measurement in the left lung is the difference in LAA between the selected lobe and the ipsilateral lobe. However, because there are 3 lobes in the right lung, the difference in LAA between the selected lobe and the 2 ipsilateral lobes is calculated by weighting the LAA in the ipsilateral lobes according to lung volume. For example, to calculate the heterogeneity in the RUL, the difference in LAA between the RUL and the LAA of the weighted ipsilateral lobe is calculated. The weighted ipsilateral lobe is calculated by summing the LAA in the RML and the RLL weighted by the volume of the corresponding lobes, and dividing by the total volume in the RML and RLL. This measurement is especially useful if the middle lobe is being considered for treatment in the indication lung volume reduction.

\section{Tissue Measurements}

Tissue Volume. Tissue volume is derived from CT density measurements because the attenuation of X-rays is directly dependent on density [40]. Tissue volume can be reported by thirds, lobes, sublobes, or by lung. A localized increase in tissue volume may indicate more a disease change in patients with lung fibrosis, interstitial pulmonary fibrosis, interstitial lung disease, or represent a change in patients with COPD disease [41].

Lung Tissue Mass. Lung tissue mass (g) is calculated from lung tissue volume. The density of tissue is $1.065 \mathrm{~g} /$ $\mathrm{mL}$ [42].

Thoracic Volume. Thoracic volume is the volume $(\mathrm{mL})$ of segmented lungs and/or lobes.

\section{Vascular Measurements}

Total Vascular Volume. Total vascular volume is the volume of all vessels (arteries and veins combined) [43, 44] (Fig. 2a). The total vascular volume is an important metric to determine vascular destruction compared to hyperinflation in patients with COPD.

Small Vessels Percent Vascular Volume. Small vessels percent vascular volume (SVPVV) is the volumetric fraction of the small vessels relative to overall vascular volume [44]. The small vessels represent the outermost layer of the airway tree and are defined using mathematical morphological erosion, not by airway generation or lumen diameter size [36]. SVPVV is a localized measure of perfusion that separates the peripheral pulmonary vessels from the central or more mediastinal pulmonary vessels. For example, SVPVV has been validated as a predictor of LVR outcome [36].

Bronchoarterial Ratio. Bronchoarterial ratio is calculated by airway segmentation at the segmental level and beyond. For a given airway segment, the corresponding adjacent artery segment is found, and the airway and ar-
Herth/Kirby/Sieren/Herth/Schirm/Wood/ Schuhmann 


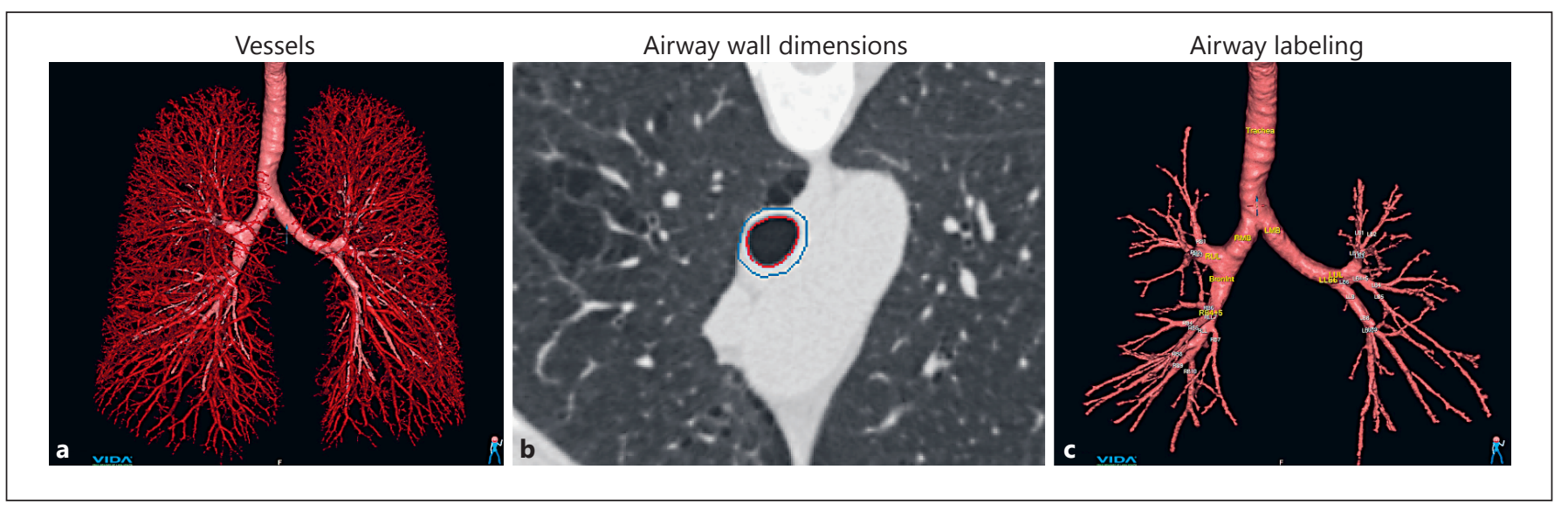

Fig. 2. QCT vessel and airway measurements. a $3 D$ representation of the vasculature (arteries and veins combined) highlighted in red (Apollo software; VIDA Diagnostics Inc., Coralville, IA, USA). b The segmentation of the airway lumen (shown in red) and the outer airway wall (shown in blue) (Apollo software). c 3D representation of the airway tree with airway labels (Apollo software).

terial diameters are measured. The bronchoarterial ratio is defined as the airway diameter divided by the accompanying arterial diameter. Healthy subjects typically have a ratio of $\sim 1: 1$ [45]. An increased ratio can, for example, occur in bronchiectasis or asthma [46]. CT-measured pulmonary artery diameter, aortic diameter, and their ratios have also been shown to be abnormal in patients with pulmonary hypertension and dyslipidemia [47].

\section{Airway Measurements}

Pi10. The Pi10 is the square root of the airway wall area for an idealized airway with the lumen perimeter of 10 $\mathrm{mm}$ [48]. Pi10 can be thought of as a standardized airway wall thickness measurement and has been shown to be significantly correlated with histological measures of the small airways [49]. Pi10 is the most commonly used measure of airway wall thickening in the literature and in many phenotyping studies of COPD and asthma, either globally (whole lung) or in a given major airway path or lobe $[48,50-52]$.

Airway Wall Thickness or Wall Fraction. The thickness of the airway wall or the fraction of the entire airway (lumen and wall) that is airway wall area, also commonly referred to as wall area percent (WA\%), can be measured as shown in Figure 2b, and are surrogate measures of airway wall remodeling. In asthma, CT airway walls have been demonstrated to be thicker compared to healthy controls $[53,54]$ and are significantly correlated with asthma duration and severity [54], airflow limitation [54, 55], and neutrophilic airway inflammation [55]. In
COPD, CT airway wall thickening has been shown to be significantly associated with lung function [56] and the frequency of exacerbations [57].

Functional Small Airway Disease. Registering CT images acquired at full inspiration to $\mathrm{CT}$ images acquired at full expiration allows lung voxels that trap gas (hyperinflated) related to tissue destruction (i.e., emphysema) to be differentiated from lung voxels that trap gas related to functional small airway disease. Two approaches have emerged that use CT inspiratory-to-expiratory methods to classify lung voxels as emphysema or functional small airway disease: parametric response map [58] and disease probability measure [59]. In COPD, functional inspiratory-to-expiratory small airway disease has been shown to be reproducible over short periods of time [60] and correlate with pulmonary function [58], and is associated with longitudinal changes in $\mathrm{FEV}_{1}$ [61].

Luminal Diameter (Max/Min). This represents the largest and the smallest diameter of an airway segment. Airway lumens have varying diameters and may not be circular, e.g. elliptical cross-section. Local disease can affect the luminal $\mathrm{max} / \mathrm{min}$ and an indicator of various disease states.

Lumen Diameter Circle Equivalent. This is the diameter of the circle with the same area as the cross-sectional area of the lumen.

Airway Circularity. Quantitative assessment of "circleness" of airway cross-section. A perfectly circular lumen has a circularity of 1 and a fully collapsed lumen has a circularity of 0 . Deformed lumens are in-between (the more deformed the closer to 0 ) [62]. Airway circularity is 
Fig. 3. QCT path planning to target. Path planning to target lesion using VIDA Diagnostics precision software (VIDA Diagnostics Inc., Coralville, IA, USA).

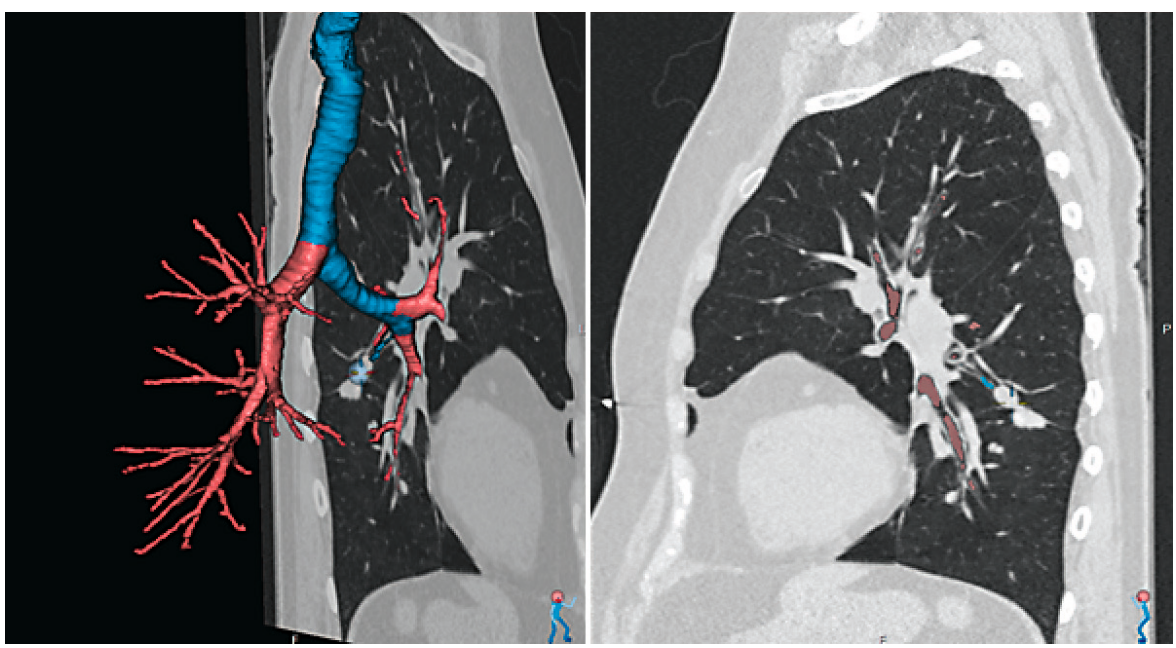

a key metric to define localized disease and fitting of devices into the airway.

Airway Concaveness. Airway concaveness expresses the severity of a cross-sectional concavity (e.g., in case of tracheomalacia) as a value between 0 (circular, no concavities) and 1 (fully concave, i.e. half-moon shape with fully collapsed lumen). Values between 0 and 1 quantify the extent of the concave area(s) relative to the overall cross-sectional area.

Airway Labeling. Airway labeling applies all 32 standard airway labels, and subsegmental labels ("a" and "b" labels in airway generations immediately following segmental airways) typically used by bronchoscopists, as shown in Figure 2c, and is based on common spatial branching patterns across populations [63]. Labeling could facilitate longitudinal comparison of airway measurements and provide a mapping or historical roadmap to a lesion.

\section{Airway Path Planning}

For pulmonologists performing transbronchial biopsies for pulmonary nodules, it has been well established that guided bronchoscopy technologies improve diagnostic yield relative to traditional transbronchial biopsy [64]. Therefore, there is motivation for developing virtual bronchoscopy planning software, such as VIDA's Lung Bronchoscopy Planning (VIDA Vision, VIDA Diagnostics Inc.), to enable path to target identification, automatic labeling of airways, and QCT measurements, as shown in Figure 3. Other bronchoscopy planning software tools are also available, such as the picture archiving and communication system (PACS), Broncus Medical
Inc., and superDimension ${ }^{\mathrm{TM}}$. PACS provides $2 \mathrm{D}$ CT images for the user to scroll through; however, there is no specialized workflow for path planning. superDimension $^{\mathrm{TM}}$ navigation system provides similar 2D planning software, with an additional real-time navigation component. The software generates 3D maps of the airways and registers patient anatomy with the $3 \mathrm{D}$ map, thereby enabling real-time navigation to show where the scope is in relation to CT. Broncus Medical Inc., provides real-time path navigation through the $3 \mathrm{D} \mathrm{CT}$ airway tree reconstruction; side-by-side navigation pairs real-time and virtual images throughout the procedure.

Angle to Target. This refers to the angle of approach vector between exit point in airway tree and target point. The angle to target is a key measure for virtual bronchoscopy to target peripheral lesions. It is important to match the specifications of the device to the architecture of the path.

Airway Extension to Target. This is the distance from the closest airway in a selected path to the target point in the lung parenchyma. The airway extension to target is a measure for virtual bronchoscopy to target peripheral lesions.

\section{Conclusions}

QCT provides anatomical and physiological insights into the structure and function of the lung in various lung diseases. QCT is now becoming an established tool to assess the pathological component of lung disease in cross-sectional and longitudinal studies providing a 
deeper understanding of how to stratify patients for treatment to improve outcomes. QCT also enables clinicians to visualize and quantify improvement following various therapeutic interventions. Furthermore, with improved image reconstruction methods that reduce ionizing radiation doses, there is potential to apply these QCT methods in longitudinal studies, and in younger patients. Respiratory physicians need to understand the different measurements and the best uses of these measurements for patient management. It is highly recommended for those who want to use QCT imaging to use standardized image acquisition protocols and quality certified and validated image analysis methodology. Further development and evaluation of CT will determine its value in the clinical management of patients with lung disease.

\section{References}

1 Mannino DM, Buist AS: Global burden of COPD: risk factors, prevalence, and future trends. Lancet 2007;370:765-773.

2 WHO: Global Surveillance, Prevention and Control of Chronic Respiratory Diseases: A Comprehensive Approach. Geneva, WHO, 2007, pp 153.

3 Rojas-Martinez R, Perez-Padilla R, Olaiz-Fernandez G, Mendoza-Alvarado L, MorenoMacias H, Fortoul T, McDonnell W, Loomis $\mathrm{D}$, Romieu I: Lung function growth in children with long-term exposure to air pollutants in Mexico City. Am J Respir Crit Care Med 2007;176:377-384.

4 Muller NL, Staples CA, Miller RR, Abboud RT: "Density mask." An objective method to quantitate emphysema using computed tomography. Chest 1988;94:782-787.

5 Dirksen A, Dijkman JH, Madsen F, Stoel B, Hutchison DC, Ulrik CS, Skovgaard LT, KokJensen A, Rudolphus A, Seersholm N, Vrooman HA, Reiber JH, Hansen NC, Heckscher T, Viskum K, Stolk J: A randomized clinical trial of alpha(1)-antitrypsin augmentation therapy. Am J Respir Crit Care Med 1999;160:1468-1472.

6 Dirksen A, Friis M, Olesen KP, Skovgaard LT, Sorensen K: Progress of emphysema in severe alpha 1-antitrypsin deficiency as assessed by annual CT. Acta Radiol 1997;38:826-832.

7 Dirksen A, Piitulainen E, Parr DG, Deng C, Wencker M, Shaker SB, Stockley RA: Exploring the role of CT densitometry: a randomised study of augmentation therapy in alphal-antitrypsin deficiency. Eur Respir J 2009;33: 1345-1353.

8 Busacker A, Newell JD Jr, Keefe T, Hoffman EA, Granroth JC, Castro M, Fain S, Wenzel S: A multivariate analysis of risk factors for the air-trapping asthmatic phenotype as measured by quantitative CT analysis. Chest 2009; 135:48-56.

9 Witt CA, Sheshadri A, Carlstrom L, Tarsi J, Kozlowski J, Wilson B, Gierada DS, Hoffman E, Fain SB, Cook-Granroth J, Sajol G, Sierra O, Giri T, O’Neill M, Zheng J, Schechtman KB, Bacharier LB, Jarjour N, Busse W, Castro M; NHLBI Severe Asthma Research Program (SARP): Longitudinal changes in airway remodeling and air trapping in severe asthma. Acad Radiol 2014;21:986-993.
10 Thomen RP, Sheshadri A, Quirk JD, Kozlowski J, Ellison HD, Szczesniak RD, Castro M, Woods JC: Regional ventilation changes in severe asthma after bronchial thermoplasty with (3)He MR imaging and CT. Radiology 2015;274:250-259.

11 Newell JD Jr, Sieren J, Hoffman EA: Development of quantitative computed tomography lung protocols. J Thorac Imaging 2013;28: 266-271.

12 Newell JD Jr, Fuld MK, Allmendinger T, Sieren JP, Chan KS, Guo J, Hoffman EA: Very low-dose $(0.15 \mathrm{mGy})$ chest CT protocols using the COPDGene 2 test object and a thirdgeneration dual-source CT scanner with corresponding third-generation iterative reconstruction software. Invest Radiol 2015;50: $40-45$.

13 Sieren JP, Hoffman EA, Fuld MK, Chan KS, Guo J, Newell JD Jr: Sinogram Affirmed Iterative Reconstruction (SAFIRE) versus weighted filtered back projection (WFBP) effects on quantitative measure in the COPDGene 2 test object. Med Phys 2014;41:091910.

14 Sieren JP, Newell JD, Judy PF, Lynch DA, Chan KS, Guo J, Hoffman EA: Reference standard and statistical model for intersite and temporal comparisons of CT attenuation in a multicenter quantitative lung study. Med Phys 2012;39:5757-5767.

15 Chen-Mayer HH, Fuld MK, Hoppel B, Judy PF, Sieren JP, Guo J, Lynch DA, Possolo A, Fain SB: Standardizing CT lung density measure across scanner manufacturers. Med Phys 2017:44:974-985.

16 Sieren JP, Newell JD Jr, Barr RG, Bleecker ER, Burnette N, Carretta EE, Couper D, Goldin J, Guo J, Han MK, Hansel NN, Kanner RE, Kazerooni EA, Martinez FJ, Rennard S, Woodruff PG, Hoffman EA; SPIROMICS Research Group: SPIROMICS protocol for multicenter quantitative computed tomography to phenotype the lungs. Am J Respir Crit Care Med 2016;194:794-806.

17 Woodruff PG, Barr RG, Bleecker E, Christenson SA, Couper D, Curtis JL, Gouskova NA, Hansel NN, Hoffman EA, Kanner RE, Kleerup E, Lazarus SC, Martinez FJ, Paine R 3rd, Rennard S, Tashkin DP, Han MK; SPIROMICS Research Group: Clinical significance of symptoms in smokers with preserved pulmonary function. N Engl J Med 2016;374: 1811-1821.

18 Gietema HA, Muller NL, Fauerbach PV, Sharma S, Edwards LD, Camp PG, Coxson HO; Evaluation of COPD Longitudinally to Identify Predictive Surrogate Endpoints (ECLIPSE) investigators: Quantifying the extent of emphysema: factors associated with radiologists' estimations and quantitative indices of emphysema severity using the ECLIPSE cohort. Acad Radiol 2011;18:661671.

19 Madani A, Zanen J, de Maertelaer V, Gevenois PA: Pulmonary emphysema: objective quantification at multi-detector row CT comparison with macroscopic and microscopic morphometry. Radiology 2006;238: 1036-1043.

20 Bankier AA, De Maertelaer V, Keyzer C, Gevenois PA: Pulmonary emphysema: subjective visual grading versus objective quantification with macroscopic morphometry and thin-section CT densitometry. Radiology 1999;211:851-858.

21 COPDGene CT Workshop Group: A combined pulmonary-radiology workshop for visual evaluation of COPD: study design, chest $\mathrm{CT}$ findings and concordance with quantitative evaluation. COPD 2012;9:151-159.

22 Cavigli E, Camiciottoli G, Diciotti S, Orlandi I, Spinelli C, Meoni E, Grassi L, Farfalla C, Pistolesi M, Falaschi F, Mascalchi M: Wholelung densitometry versus visual assessment of emphysema. Eur Radiol 2009;19:16861692.

23 Lederer DJ, Enright PL, Kawut SM, Hoffman EA, Hunninghake G, van Beek EJ, Austin JH, Jiang R, Lovasi GS, Barr RG: Cigarette smoking is associated with subclinical parenchymal lung disease: the Multi-Ethnic Study of Atherosclerosis (MESA)-lung study. Am J Respir Crit Care Med 2009;180:407-414.

24 Kliment CR, Araki T, Doyle TJ, Gao W, Dupuis J, Latourelle JC, Zazueta OE, Fernandez IE, Nishino M, Okajima Y, Ross JC, Estepar RS, Diaz AA, Lederer DJ, Schwartz DA, Silverman EK, Rosas IO, Washko GR, O'Connor GT, Hatabu H, Hunninghake GM: A comparison of visual and quantitative methods to identify interstitial lung abnormalities. BMC Pulm Med 2015;15:134. 
25 Podolanczuk AJ, Oelsner EC, Barr RG, Hoffman EA, Armstrong HF, Austin JH, Basner RC, Bartels MN, Christie JD, Enright PL, Gochuico BR, Hinckley Stukovsky K, Kaufman JD, Hrudaya Nath P, Newell JD Jr, Palmer SM, Rabinowitz D, Raghu G, Sell JL, Sieren J, Sonavane SK, Tracy RP, Watts JR, Williams K, Kawut SM, Lederer DJ: High attenuation areas on chest computed tomography in community-dwelling adults: the MESA study. Eur Respir J 2016;48:1442-1452.

26 Mishima M, Hirai T, Itoh H, Nakano Y, Sakai H, Muro S, Nishimura K, Oku Y, Chin K, Ohi M, Nakamura T, Bates JH, Alencar AM, Suki B: Complexity of terminal airspace geometry assessed by lung computed tomography in normal subjects and patients with chronic obstructive pulmonary disease. Proc Natl Acad Sci USA 1999;96:8829-8834.

27 Gould GA, MacNee W, McLean A, Warren PM, Redpath A, Best JJ, Lamb D, Flenley DC: CT measurements of lung density in life can quantitate distal airspace enlargement - an essential defining feature of human emphysema. Am Rev Respir Dis 1988;137:380-392.

28 Parr DG, Sevenoaks M, Deng C, Stoel BC, Stockley RA: Detection of emphysema progression in alpha 1-antitrypsin deficiency using CT densitometry; methodological advances. Respir Res 2008;9:21.

29 Parr DG, Stoel BC, Stolk J, Stockley RA: Validation of computed tomographic lung densitometry for monitoring emphysema in alpha1-antitrypsin deficiency. Thorax 2006;61: 485-490.

30 Jain N, Covar RA, Gleason MC, Newell JD Jr, Gelfand EW, Spahn JD: Quantitative computed tomography detects peripheral airway disease in asthmatic children. Pediatr Pulmonol 2005; 40:211-218.

31 Newman KB, Lynch DA, Newman LS, Ellegood D, Newell JD Jr: Quantitative computed tomography detects air trapping due to asthma. Chest 1994;106:105-109.

32 Schroeder JD, McKenzie AS, Zach JA, Wilson CG, Curran-Everett D, Stinson DS, Newell JD Jr, Lynch DA: Relationships between airflow obstruction and quantitative CT measurements of emphysema, air trapping, and airways in subjects with and without chronic obstructive pulmonary disease. AJR Am J Roentgenol 2013;201:W460-W470.

33 Ueda T, Niimi A, Matsumoto H, Takemura M, Hirai T, Yamaguchi M, Matsuoka H, Jinnai M, Muro S, Chin K, Mishima M: Role of small airways in asthma: investigation using high-resolution computed tomography. J Allergy Clin Immunol 2006;118:1019-1025.

34 Tunon-de-Lara JM, Laurent F, Giraud V, Perez T, Aguilaniu B, Meziane H, Basset-Merle A, Chanez P: Air trapping in mild and moderate asthma: effect of inhaled corticosteroids. J Allergy Clin Immunol 2007;119:583-590.
35 Newell JD, Raffy P: Automatic quantification of lung fissure integrity in subjects with severe emphysema; C77 Imaging: Physiologic and Clinical Correlates, Emerging Technologies. ATS Int Conf, Philadelphia, May 2013, pp A4897-A4897.

36 Schuhmann M, Raffy P, Yin Y, Gompelmann D, Oguz I, Eberhardt R, Hornberg D, Heussel CP, Wood S, Herth FJ: Computed tomography predictors of response to endobronchial valve lung reduction treatment. Comparison with Chartis. Am J Respir Crit Care Med 2015;191:767-774.

37 McKenna RJ Jr, Brenner M, Fischel RJ, Singh N, Yoong B, Gelb AF, Osann KE: Patient selection criteria for lung volume reduction surgery. J Thorac Cardiovasc Surg 1997;114: 957-964; discussion 964-957.

38 Sciurba FC, Ernst A, Herth FJ, Strange C, Criner GJ, Marquette CH, Kovitz KL, Chiacchierini RP, Goldin J, McLennan G; VENT Study Research Group: A randomized study of endobronchial valves for advanced emphysema. N Engl J Med 2010;363:1233-1244.

39 Herth FJ, Noppen M, Valipour A, Leroy S, Vergnon JM, Ficker JH, Egan JJ, Gasparini S, Agusti C, Holmes-Higgin D, Ernst A; International VENT Study Group: Efficacy predictors of lung volume reduction with Zephyr valves in a European cohort. Eur Respir J 2012;39:1334-1342.

40 Coxson HO, Mayo JR, Behzad H, Moore BJ, Verburgt LM, Staples CA, Pare PD, Hogg JC: Measurement of lung expansion with computed tomography and comparison with quantitative histology. J Appl Physiol (1985) 1995;79:1525-1530.

41 Barjaktarevic I, Springmeyer S, Gonzalez X, Sirokman W, Coxson HO, Cooper CB: Diffusing capacity for carbon monoxide correlates best with tissue volume from quantitative CT scanning analysis. Chest 2015;147: 1485-1493.

42 Hogg JC, Nepszy S: Regional lung volume and pleural pressure gradient estimated from lung density in dogs. J Appl Physiol 1969;27:198203.

43 Shikata H, Hoffman EA, Sonka M: Automated segmentation of pulmonary vascular tree from 3D CT images. Proc SPIE 5369, Medical Imaging 2004: Physiology, Function, and Structure from Medical Images (April 30, 2004); DOI: $10.1117 / 12.537032$.

44 Shikata H, McLennan G, Hoffman EA, Sonka M: Segmentation of pulmonary vascular trees from thoracic 3D CT images. Int J Biomed Imaging 2009;2009:636240.

45 Kim SJ, Im JG, Kim IO, Cho ST, Cha SH, Park KS, Kim DY: Normal bronchial and pulmonary arterial diameters measured by thin section CT. J Comput Assist Tomogr 1995;19:365-369.

46 Matsuoka S, Uchiyama K, Shima H, Ueno N, Oish S, Nojiri Y: Bronchoarterial ratio and bronchial wall thickness on high-resolution CT in asymptomatic subjects: correlation with age and smoking. AJR Am J Roentgenol 2003; 180:513-518.
47 Lee SH, Kim YJ, Lee HJ, Kim HY, Kang YA, Park MS, Kim YS, Kim SK, Chang J, Jung JY: Comparison of CT-determined pulmonary artery diameter, aortic diameter, and their ratio in healthy and diverse clinical conditions. PLoS One 2015; 10:e0126646.

48 Grydeland TB, Dirksen A, Coxson HO, Pillai SG, Sharma S, Eide GE, Gulsvik A, Bakke PS: Quantitative computed tomography: emphysema and airway wall thickness by sex, age and smoking. Eur Respir J 2009;34:858-865.

49 Nakano Y, Wong JC, de Jong PA, Buzatu L, Nagao T, Coxson HO, Elliott WM, Hogg JC, Pare PD: The prediction of small airway dimensions using computed tomography. Am J Respir Crit Care Med 2005;171:142-146.

50 Patel BD, Coxson HO, Pillai SG, Agusti AG, Calverley PM, Donner CF, Make BJ, Muller NL, Rennard SI, Vestbo J, Wouters EF, Hiorns MP, Nakano Y, Camp PG, Nasute Fauerbach PV, Screaton NJ, Campbell EJ, Anderson WH, Pare PD, Levy RD, Lake SL, Silverman EK, Lomas DA; International COPD Genetics Network: Airway wall thickening and emphysema show independent familial aggregation in chronic obstructive pulmonary disease. Am J Respir Crit Care Med 2008;178:500505.

51 Smith BM, Hoffman EA, Rabinowitz D, Bleecker E, Christenson S, Couper D, Donohue KM, Han MK, Hansel NN, Kanner RE, Kleerup E, Rennard S, Barr RG: Comparison of spatially matched airways reveals thinner airway walls in COPD. The Multi-Ethnic Study of Atherosclerosis (MESA) COPD Study and the Subpopulations and Intermediate Outcomes in COPD Study (SPIROMICS). Thorax 2014;69:987-996.

52 Woodruff PG, Couper D, Han MK: Symptoms in smokers with preserved pulmonary function. N Engl J Med 2016;375:896-897.

53 Grenier P, Mourey-Gerosa I, Benali K, Brauner MW, Leung AN, Lenoir S, Cordeau MP, Mazoyer B: Abnormalities of the airways and lung parenchyma in asthmatics: CT observations in 50 patients and inter- and intraobserver variability. Eur Radiol 1996;6:199-206.

54 Niimi A, Matsumoto H, Amitani R, Nakano Y, Mishima M, Minakuchi M, Nishimura K, Itoh $\mathrm{H}$, Izumi T: Airway wall thickness in asthma assessed by computed tomography. Relation to clinical indices. Am J Respir Crit Care Med 2000;162:1518-1523.

55 Gupta S, Siddiqui S, Haldar P, Entwisle JJ, Mawby D, Wardlaw AJ, Bradding P, Pavord ID, Green RH, Brightling CE: Quantitative analysis of high-resolution computed tomography scans in severe asthma subphenotypes. Thorax 2010;65:775-781.

56 Nakano Y, Muro S, Sakai H, Hirai T, Chin K, Tsukino M, Nishimura K, Itoh H, Pare PD, Hogg JC, Mishima M: Computed tomographic measurements of airway dimensions and emphysema in smokers. Correlation with lung function. Am J Respir Crit Care Med 2000;162:1102-1108.

Herth/Kirby/Sieren/Herth/Schirm/Wood/ Schuhmann 
57 Han MK, Kazerooni EA, Lynch DA, Liu LX, Murray S, Curtis JL, Criner GJ, Kim V, Bowler RP, Hanania NA, Anzueto AR, Make BJ, Hokanson JE, Crapo JD, Silverman EK, Martinez FJ, Washko GR; COPDGene Investigators: Chronic obstructive pulmonary disease exacerbations in the COPDGene study: associated radiologic phenotypes. Radiology 2011;261:274-282.

58 Galban CJ, Han MK, Boes JL, Chughtai KA, Meyer CR, Johnson TD, Galban S, Rehemtulla A, Kazerooni EA, Martinez FJ, Ross BD: Computed tomography-based biomarker provides unique signature for diagnosis of COPD phenotypes and disease progression. Nat Med 2012;18:1711-1715.

59 Kirby M, Tan WC, Bourbeau J, Hogg JC, Yin Y, Coxson HO: Novel computed tomography disease probability measure analysis technique provides sensitive imaging biomarkers of chronic obstructive pulmonary disease phenotypes in subjects with mild-to-moderate disease; Radiol Soc North Am 2014 Sci Assembly Annu Meet, Chicago, 2014.

60 Boes JL, Hoff BA, Bule M, Johnson TD, Rehemtulla A, Chamberlain R, Hoffman EA, Kazerooni EA, Martinez FJ, Han MK, Ross BD, Galban CJ: Parametric response mapping monitors temporal changes on lung CT scans in the subpopulations and intermediate outcome measures in COPD Study (SPIROMICS). Acad Radiol 2015;22:186-194.

61 Bhatt SP, Soler X, Wang X, Murray S, Anzueto AR, Beaty TH, Boriek AM, Casaburi R, Criner GJ, Diaz AA, Dransfield MT, Curran-Everett D, Galban CJ, Hoffman EA, Hogg JC, Kazerooni EA, Kim V, Kinney GL, Lagstein A, Lynch DA, Make BJ, Martinez FJ, Ramsdell JW, Reddy R, Ross BD, Rossiter HB, Steiner RM, Strand MJ, van Beek EJ, Wan ES, Washko GR,
Wells JM, Wendt CH, Wise RA, Silverman EK, Crapo JD, Bowler RP, Han MK; COPDGene Investigators: Association between functional small airway disease and FEV1 decline in chronic obstructive pulmonary disease. Am J Respir Crit Care Med 2016;194:178-184.

62 Choi S, Hoffman EA, Wenzel SE, Castro M, Fain SB, Jarjour NN, Schiebler ML, Chen K, Lin CL: Quantitative assessment of multiscale structural and functional alterations in asthmatic populations. J Appl Physiol (1985) 2015;118:1286-1298.

63 Tschirren J, McLennan G, Palágyi K, Hoffman EA, Sonka M: Matching and anatomical labeling of human airway tree. IEEE Trans Med Imaging 2005;24:1540-1547.

64 Wang Memoli JS, Nietert PJ, Silvestri GA: Meta-analysis of guided bronchoscopy for the evaluation of the pulmonary nodule. Chest 2012;142:385-393. 